\title{
CARACTERIZACIÓN PRODUCTIVA DE SISTEMAS DE CRÍA BOVINA TECNIFICADOS DE LA REGIÓN CENTRO NORTE DE ARGENTINA
}

\author{
PRODUCTIVE CHARACTERIZATION OF TECHNIFIED COW-CALF \\ SYSTEMS OF THE NORTH-CENTRAL REGION OF ARGENTINA
}

\author{
Guillermina Gregoretti ${ }^{*}$, Javier Baudracco ${ }^{1}$, Carlos Dimundo ${ }^{2,3}$, Agustín Alesso ${ }^{1}$, Belén Lazzarini ${ }^{1}$ y \\ Claudio Machado ${ }^{4}$

\begin{abstract}
${ }^{1}$ IciAgro Litoral, Universidad Nacional del Litoral, Consejo Nacional de Investigaciones Científicas y Técnicas (CONICET), Facultad de Ciencias Agrarias, Kreder 2805, Esperanza, CP: S3080HOF, Argentina.

${ }^{2}$ Facultad de Ciencias Agrarias, Universidad Nacional del Litoral, Kreder 2805, Esperanza, CP: S3080HOF, Argentina.

${ }^{3}$ CREA Región Norte de Santa Fe, Asociación Argentina de Consorcios Regionales de Experimentación Agrícola (AACREA), Sarmiento 1236, Buenos Aires, CP: C1041AAZ, Argentina.

${ }^{4}$ CIVETAN (Facultad de Ciencias Veterinarias, CIC, CONICET), Paraje Arroyo Seco s/n, Campus Universitario, Tandil, CP: B7000GHG, Argentina.

*Autor para correspondencia: guillerminagregoretti.gg@gmail.com
\end{abstract}

\section{RESUMEN}

La caracterización productiva de sistemas ganaderos es clave para diseñar planes de mejora en sistemas de producción de carne de una región. El objetivo de este trabajo fue identificar características productivas de sistemas de cría tecnificados del norte de la provincia de Santa Fe en Argentina, y analizar la relación entre las variables identificadas y su efecto sobre la producción de carne. Se relevaron 16 variables de estructura, tecnológicas y de eficiencia productiva y reproductiva en 27 establecimientos. Se realizaron análisis del tipo descriptivo y multivariado, con un nivel de significancia $\mathrm{p} \leq 0,05$. La carga animal (vacas $\mathrm{ha}^{-1}$ ), el porcentaje de destete y la producción de carne (kg PV ha ${ }^{-1}$ año $^{-1}$ ) de los sistemas tecnificados evaluados fueron un 15, 38 y $84 \%$ superiores, respectivamente, respecto a sistemas típicos de la región. La mayor superficie con especies cultivadas se correlacionó positivamente con los porcentajes de preñez $(p=0,0269)$ y destete $(p=0,005)$. El nivel de suplementación de las vacas $(\mathrm{p}=0,0259)$ se asoció positivamente con la carga animal $\left(\right.$ vacas $\left.\mathrm{ha}^{-1}\right)$. A su vez, la carga animal (vacas ha-1) correlacionó positivamente con una mayor producción de terneros (kg PV ha-1 año $\left.{ }^{-1} ; \mathrm{p}<0,0001\right)$ y producción de carne total (kg PV ha $\left.{ }^{-1} \mathrm{año}^{-1} ; \mathrm{p}<0,0001\right)$. Los predios tecnificados presentaron mejores indicadores productivos que los predios de cría típicos de la región estudiada.

Palabras clave: producción de carne, suplementación, carga animal, sistemas.

\section{ABSTRACT}

The productive characterization of livestock systems is a key aspect when designing management plans aimed at increasing beef production in a region. The objective of this work was to characterize technified cow-calf systems of the north region of the Santa Fe province, Argentina, and to analyze the correlation between the variables identified and the effect on beef production. Sixteen variables related to structure, technology, and productive and reproductive efficiency were assessed in 27

Recibido: 27 julio $2020 . \quad$ Aceptado: 19 octubre 2020. 
farms. Descriptive statistics and multivariate analyzes were performed at a significance level of $p$ $\leq 0.05$. Stocking rate (cows ha-1), weaning rate $(\%)$ and beef production $\left(\mathrm{kg} \mathrm{LW} \mathrm{ha-1} \mathrm{year}^{-1}\right)$ of the surveyed farms were 15,38 and $84 \%$ higher, respectively, compared with typical systems of the region. The higher area with cultivated species was positively correlated with pregnancy rate ( $p$ $=0.0269)$ and weaning rate $(p=0.0051)$. Supplementation level of cows $(p=0.0259)$ was positively associated with stocking rate (cows ha-1). Furthermore, stocking rate (cows ha-1) correlated positively with higher calf production $\left(\mathrm{kg} \mathrm{LW} \mathrm{ha}^{-1}\right.$ year $\left.{ }^{-1} ; \mathrm{p}<0.0001\right)$ and total beef production $(\mathrm{kg} \mathrm{LW} \mathrm{ha-1}$ year $\left.{ }^{1} ; \mathrm{p}<0.0001\right)$. Technified farms showed a better productive performance compared to that of typical cow-calf systems in the region studied.

Key words: beef production, supplementation, stocking rate, systems.

\section{INTRODUCCIÓN}

La producción de carne bovina contribuye al desarrollo económico y social en los países y es una parte importante de la dieta humana (Pighin et al., 2016). Según la FAO (2019), se requerirá $50 \%$ más de alimento para el año 2050 debido al incremento de la población mundial. Esto deberá lograrse aumentando la eficiencia productiva de los sistemas, ya que se proyecta una menor disponibilidad de tierra cultivable para la producción de alimentos para el ganado (Britt et al., 2018).

En Argentina, el porcentaje de destete y el peso de faena nacional han permanecido relativamente estable en los últimos 30 años, con valores promedio de $60 \%$ y $225 \mathrm{~kg}$, respectivamente (MAGyP, 2019). Estos valores se consideran bajos cuando se los compara con otros países productores de carne, como Australia, que tiene un promedio de $77 \%$ de destete anual (USDA, 2019) y peso promedio de faena de $270 \mathrm{~kg}$ (Meat and Livestock Australia, 2017). Además, en las últimas décadas, los sistemas de cría en Argentina se han concentrado en suelos de menor aptitud forrajera (Rearte y Pordomingo, 2014), debido a un avance de la producción de granos en superficies ganaderas, con el agravante que estos sistemas en general presentan baja adopción de tecnologías, como el establecimiento de praderas, la fertilización y la suplementación (Rearte y Pordomingo, 2014).

La provincia de Santa Fe, ubicada en el centronorte de Argentina, es la segunda productora de carne bovina a nivel nacional, después de Buenos Aires. Tiene un stock de 6 millones de cabezas y produce el $10 \%$ de los terneros del país y el $17 \%$ de la producción de carne nacional (MAGyP, 2019). Aproximadamente $60 \%$ de la existencia bovina provincial se concentra en la región norte de la provincia (MAGyP, 2019), y la actividad ganadera principal es la cría bovina sobre campo natural. En efecto, aproximadamente $40 \%$ de los productores de cría de la provincia se concentran en esa región (Chimicz, 2006). Los sistemas de esta región presentan baja adopción de tecnología de insumos y tienen bajos índices productivos $\left(0,4\right.$ vacas ha- ${ }^{-1}, 50 \%$ de destete y 45 kg de carne ha ${ }^{-1}$ año $^{-1}$ (Chimicz, 2006; Chiossone, 2006; CNA, 2018), en concordancia con la mayoría de los sistemas del país. Sin embargo, existen en la región algunos productores agrupados, que reciben asesorías y que aplican tecnologías y obtienen mejores niveles de eficiencia que los sistemas promedio, sobre los cuales no se ha publicado información.

La caracterización productiva de sistemas ganaderos de una región es clave para identificar, evaluary promocionar potenciales oportunidades de mejoras para sistemas con índices productivos inferiores (Faverin et al., 2019; Fernandez Rosso et al., 2020). Dicha caracterización puede realizarse a partir de datos cuantitativos provenientes de relevamientos (Faverin y Machado, 2019; Lara et al., 2019), complementándose con la opinión de expertos, la cual permite caracterizar de manera integral sistemas complejos y dinámicos como los sistemas agropecuarios (Kuivanen et al., 2016). Por lo tanto, el objetivo de este trabajo fue caracterizar, mediante un relevamiento los sistemas de cría tecnificados del norte de Santa $\mathrm{Fe}$, y evaluar la relación entre las variables relevadas y su efecto sobre la producción de carne, y de este modo identificar oportunidades para otros productores de la región.

\section{MATERIALES Y MÉTODOS}

\section{Área de estudio}

El área de estudio está situada entre los paralelos 28 a $30^{\circ}$ Sur y los meridianos 62 a $59^{\circ}$ Oeste, tiene aproximadamente 5 millones de hectáreas de uso agropecuario (CNA, 2008) e incluye los departamentos Nueve de Julio, Vera, General Obligado y el norte de los departamentos San Cristóbal, San Justo y San Javier de la provincia de Santa Fe, Argentina. La actividad ganadera principal de esta región es la cría extensiva sobre campo natural (IPCVA, 2016). El clima es templado-cálido y húmedo con un 
gradiente de precipitación media anual entre 975 $\mathrm{mm}$ y $1.200 \mathrm{~mm}$, desde el oeste al este de la región, concentrada en la estación cálida (octubre a abril). La temperatura media anual es $20,1^{\circ} \mathrm{C}$ con una mínima media de $13,9^{\circ} \mathrm{C}$ y una máxima media de $26,2^{\circ} \mathrm{C}$, en julio y enero, respectivamente (INTA, 2018). El período libre de heladas es de 220 a 260 días (INTA, 2018). Predominan suelos sódicos y salinos-sódicos con drenaje deficiente (Giorgi, 2007).

\section{Relevamiento}

La caracterización de sistemas de cría tecnificados de la región se realizó a partir de un relevamiento de todos los establecimientos $(\mathrm{n}=27)$ pertenecientes a una asociación no gubernamental CREA (Asociación Argentina de Consorcios Regionales de Experimentación Agropecuaria, www.crea.org.ar) en la región bajo estudio. Esta asociación nuclea a productores que trabajan en grupos de discusión con asesores técnicos y presentan mayor adopción tecnológica y habilidad de gestión de recursos en comparación con productores típicos de la zona (Pacin y Oesterheld, 2015; Gallacher y Lema, 2018). Los establecimientos relevados de cría bovina presentan registros productivos, y cuentan con asesoramiento agronómico permanente. El relevamiento realizado incluyó 37 preguntas que se recopilaron a través de los asesores del grupo (9 en total), quienes recibieron la encuesta por correo electrónico y respondieron el cuestionario utilizando datos de los registros productivos de un año (julio 2016 a junio 2017). Luego de recopilar y analizar las respuestas se realizó un taller con los asesores para validar las variables relevadas. Las variables relevadas (16) se agruparon en variables de estructura, tecnológicas $\mathrm{y}$ de eficiencia productiva $\mathrm{y}$ reproductiva.

Variables de estructura. Superficie de cría (ha, incluye las hectáreas destinadas a vacas, toros y vaquillonas), régimen de tenencia de la tierra (\% de superficie de cría propia), existencia de vacas (cabezas), de toros (\% sobre existencias de vacas), existencia de vaquillonas (\% sobre existencias vacas)

Variables tecnológicas. Superficie cultivada (\% de la superficie de cría con especies cultivadas), niveles de suplementación de las categorías (\% del peso vivo), duración periodo de encaste (meses), edad de primer servicio (meses) y edad de las crías al destete (meses).

Variables de eficiencia productiva $y$ reproductiva. Carga animal (vacas ha ${ }^{-1}$ ), preñez $(\%$, vientres preñados sobre el total de vientres encastados), destete (\%, terneros destetados sobre el total de vientres encastados), producción de terneros $\left(\mathrm{kg} \mathrm{PV} \mathrm{ha-1} \mathrm{año}{ }^{-1}\right)$ y producción de carne total ( $\mathrm{kg} \mathrm{PV} \mathrm{ha}^{-1}$ año-1), estimados según lo propuesto por Canosa (2003).

\section{Análisis estadístico}

Se realizó un análisis descriptivo (promedio, desvío estándar y valores máximos (MAX) y mínimos (MIN) para caracterizar cada una de las variables relevadas. Un análisis de componentes principales y de forma complementaria correlaciones de Pearson (con nivel de significancia $\mathrm{p} \leq 0,05)$. Adicionalmente, se realizó un análisis de agrupamiento para identificar grupos de establecimientos con similares características productivas, e identificar estrategias de manejo diferentes entre grupos. Se utilizó el método de agrupamiento jerárquico a partir de distancias Euclídeas estandarizadas y método de Ward (Ward, 1963). Los resultados se presentaron mediante el dendrograma. Para la realización de todos los análisis se utilizó el software Infostat versión 2018 (Di Rienzo et al., 2011).

\section{RESULTADOS}

\section{Caracterización productiva de los predios relevados}

Variables de estructura. La superficie de cría, el promedio de vacas y la carga animal promedio fueron $1.801 \pm 1.883$ ha, $828 \pm 360$ cabezas, y 0,46 \pm 0,20 vacas ha $^{-1}$, respectivamente (Tabla 1 ).

Variables tecnológicas. En promedio, 90\% de la superficie relevada estuvo ocupada por campo natural con especies nativas no sembradas tanto gramíneas C3 (Leersia hexandra Sw., Luziola peruviana Juss. Ex J.F. Gmel., Bromus catharticus Vahl, y varias especies del género Nacella, Hordeum y Panicum.), como gramíneas C4 (Andropogon lateralis Nees, Sorghastrum setosum Hitchc, y especies de los géneros Setaria, Echinochloa, Pappophorum y Paspalum, este último representado por varias especies), leguminosas forrajeras (especies de los géneros Desmodium, Desmanthus y Vicia) y áreas boscosas con arbustos y árboles en su mayoría leguminosas, de follaje ramoneables y frutos forrajeros (varias especies del género Prosopis). El 10\% restante de la superficie relevada estuvo ocupada por especies cultivadas (Avena sativa L., Zea mays L., Sorghum bicolor L. Moench, y Chloris gayana K.). Por otro lado, menos de la mitad de los predios relevados $(44 \%)$ sembraron $3 \pm 5 \%$ y $3 \pm 6 \%$ de la superficie total con verdeos (cultivos anuales) de invierno y de verano, respectivamente. Además, un $37 \%$ de estos sembraron praderas megatérmicas perennes Chloris gayana K., y en menor medida algunas especies del género Brachiaria, en $7 \pm 10 \%$ de la superficie total, y utilizaron niveles de fertilización 
Tabla 1. Valores promedio, desvío estándar (DE), valores máximos (MAX) y mínimos (MIN) de las variables de estructura, tecnológicas y de eficiencia productiva y reproductiva en los sistemas de cría relevados $(\mathrm{n}=27)$.

Table 1. Mean values, standard deviation (SD), and maximum (MAX) and minimum (MIN) values for the structural, technological, and productive and reproductive efficiency variables of the cow-calf systems surveyed $(n=27)$.

\begin{tabular}{llcrrr}
\hline Variables & Unidad & Promedio & DE & MAX & MIN \\
\hline De estructura & & & & & \\
Superficie de cría & ha totales & 1.801 & 1.883 & 9.058 & 176 \\
Superficie de cría propia & \% de la superficie de cría & 77 & 36 & 100 & 10 \\
Existencia de toros & \% sobre existencia de vacas & 4 & 1 & 8 & 2 \\
Existencia de vaquillonas & \% sobre existencia de vacas & 20 & 5 & 25 & 15 \\
\hline Tecnológicas & & & & & \\
Superficie con & & & & & \\
especies cultivadas & \% de la superficie de cría & 10 & 11 & 35 & 0 \\
Suplementación vaquillonas & \% del peso vivo & 0,83 & 0,54 & 1,50 & 0 \\
Suplementación vacas & \% del peso vivo & 0,15 & 0,30 & 1,00 & 0 \\
Suplementación toros & \% del peso vivo & 0,74 & 0,42 & 1,50 & 0 \\
Edad de primer servicio & Meses & 23 & 4 & 27 & 12 \\
Duración del servicio & Meses & 4 & 1 & 7 & 3 \\
Edad del destete & Meses & 6 & 2 & 8 & 4 \\
\hline Eficiencia productiva y & & & & & \\
reproductiva & & & & & \\
Carga animal & \% & 0,46 & 0,20 & 0,85 & 0,21 \\
Preñez & \% & 69 & 9 & 85 & 50 \\
Destete & kg PV ha haño $^{-1}$ & 51 & 26 & 112 & 43 \\
Producción de terneros & kg PV ha año $^{-1}$ & 83 & 37 & 153 & 33 \\
Producción de carne & & & & \\
\hline
\end{tabular}

nitrogenada inferiores a $25 \mathrm{~kg}$ de $\mathrm{N} \mathrm{ha}^{-1}$ año ${ }^{1}$.

La suplementación con concentrados y ensilaje es frecuente en vaquillonas de reposición (67 y $42 \%$ de los casos, respectivamente; Tabla 2). Con respecto a la duración del servicio, $60 \%$ de los predios concentraron los servicios durante 4 meses, y $78 \%$ de estos comienzan en los meses de octubre y noviembre. La edad al primer servicio es de $23 \pm 4$ meses (Tabla 1 ). El destete se realiza principalmente en el mes de marzo con una edad promedio de la cría de $6 \pm 2$ meses.

Eficiencia productiva y reproductiva. Los porcentajes de preñez y destete fueron $77 \pm 9$ y $69 \pm 9 \%$, respectivamente (Tabla 1). Los terneros fueron destetados con $153 \pm 23 \mathrm{~kg}$ y las vacas de descarte destinadas a venta pesaron $460 \pm 39 \mathrm{~kg}$ de PV. La productividad de terneros y de carne fue $51 \pm 26$ y $83 \pm 37$ de kg ha $^{-1}$ año $^{-1}$, respectivamente (Tabla 1).

\section{Análisis de componentes principales}

El $31,1 \%$ de la variación de los datos está explicada por la primera componente (CP1)
(Fig. 1), asociada al nivel de suplementación, porcentaje de superficie cultivada (verdeos y praderas), preñez (\%), destete (\%), la producción de carne ( $\mathrm{kg}$ PV ha-1 por año) y la carga animal (vacas ha-1). La segunda componente principal (CP2) explica el $18,0 \%$ de la variación y se relaciona con la duración del servicio (meses) y la edad al destete en meses (Fig. 1).

\section{Análisis de correlaciones}

En la Tabla 3 se presentan los coeficientes de correlación de Pearson y el nivel de significancia solamente para las correlaciones que fueron estadísticamente significativas $(\mathrm{p}<0,05$ y $\mathrm{p}<$ 0,01 . Acorde a lo detectado en el análisis de componentes principales, cuanto mayor fue el porcentaje de superficie destinada a verdeos anuales y praderas megatérmicas, mayores fueron los porcentajes de preñez y destete (Tabla 3). Del mismo modo, la carga animal (vientres ha $^{-1}$ ) se asoció positivamente con los niveles de suplementación, producción por ha y por año de terneros y producción de carne total (Tabla 3). 
Tabla 2. Porcentaje de los predios relevados $(n=27)$ que realizan suplementación, suplementos utilizados y momento de suplementación. Los porcentajes de suplementación se presentan por categorías: vacas, hembras para reposición y toros, y se expresan como predios que suplementan sobre predios totales $(n=27) \times 100$.

Table 2. Percentage of surveyed farms $(n=27)$ that use supplementation, type of supplements and time of supplementation. Supplementation rates are presented by category: cows, heifers and bulls, and expressed as farms that perform supplementation with respect to the total number of farms $(n=27) \times 100$.

\begin{tabular}{lccc}
\hline Categoría & Vacas & $\begin{array}{c}\text { Hembras para } \\
\text { reposición }\end{array}$ & Toros \\
\hline Predios que suplementan (\% de casos) & 26 & 89 & 89 \\
Suplementos utilizados & 43 & 42 & 13 \\
Ensilaje de verdeos anuales (\% de casos) & 29 & 38 & 17 \\
Heno (\% de casos) & & & \\
Concentrado energético-proteico & 29 & 67 & 33 \\
$\quad$ (\% de casos) & - & 21 & 21 \\
Concentrado energético (\% de casos) & - & 29 & 17 \\
Concentrado proteico (\% de casos) & 14 & 4 & 4 \\
Cáscaras (\% de casos) & 14 & 8 & - \\
Uso de diferidos (\% de casos) & & & 42 \\
Momento de suplementación & 83 & - & - \\
Invierno (\% de casos) & - & 21 & - \\
Primavera (\% de casos) & 17 & 17 & - \\
Otoño-Invierno (\% de casos) & - & 4 & \\
Invierno-Primavera (\% de casos) & - & & \\
Otoño-Invierno-Primavera (\% de casos) & - & & \\
Todo el año (\% de casos) & & 29 & \\
\hline
\end{tabular}

\section{Análisis de conglomerados}

El análisis de conglomerados permitió identificar tres grupos de establecimientos (Fig. 2) cuyos valores promedio y desvíos estándar para cada variable relevada se muestra en la Tabla 4 . El grupo 1 conformado por 15 establecimientos presentó menor producción de carne que el resto de los grupos $\left(62 \pm 24 \mathrm{~kg}\right.$ PV ha- $\left.{ }^{-1} \mathrm{año}^{-1}\right)$. En el grupo 1 la carga animal (vientres ha-1) fue más baja que en los demás grupos, y presentó menor superficie con especies cultivadas (\% de la superficie de cría) y bajo nivel de suplementación de las vacas (\% del peso vivo). En contraste, los grupos 2 y 3 presentaron mayor producción de carne que el grupo 1. El grupo 2, conformado por 8 establecimientos, fue el grupo con mayor producción de carne $\left(116 \pm 35 \mathrm{~kg} \mathrm{PV} \mathrm{ha}^{-1} \mathrm{año}^{-1}\right)$, la cual estuvo asociada principalmente a una mayor carga animal (vientres ha $\mathrm{a}^{-1}$ ) y mayor nivel de suplementación de las vacas (\% del peso vivo) respecto al grupo 1 (Tabla 4). El grupo 3, conformado por 4 establecimientos, presentó una producción de carne levemente inferior al grupo $2\left(97 \pm 33 \mathrm{~kg} \mathrm{PV} \mathrm{ha}^{-1}\right.$ año $\left.^{-1}\right)$. Las principales variables que explican la mayor producción de carne en el grupo 3 respecto al grupo 1 fueron mayor superficie cultivada (\% de la superficie de cría), mayor preñez (\%) y mayor destete (\%) (Tabla 4).

\section{DISCUSIÓN}

La información obtenida en este trabajo permite caracterizar productivamente sistemas tecnificados del norte de Santa Fe (Tabla 1), que presentan 84 y 15\% mayor producción de carne y carga animal que los sistemas típicos de la misma región (Chimicz, 2006; Chiossone, 2006). Lo mismo se observó para la variable porcentaje de destete (Tabla 1), que resultó 38\% mayor que el valor reportado en los sistemas típicos de la región (Capozzolo et al., 2017; MAGyP, 2019). Las causas del bajo porcentaje de destete de sistemas típicos de la región son múltiples, entre los que se nombra un manejo deficiente del plan sanitario (Abdala et al., 2013), falta de adopción 


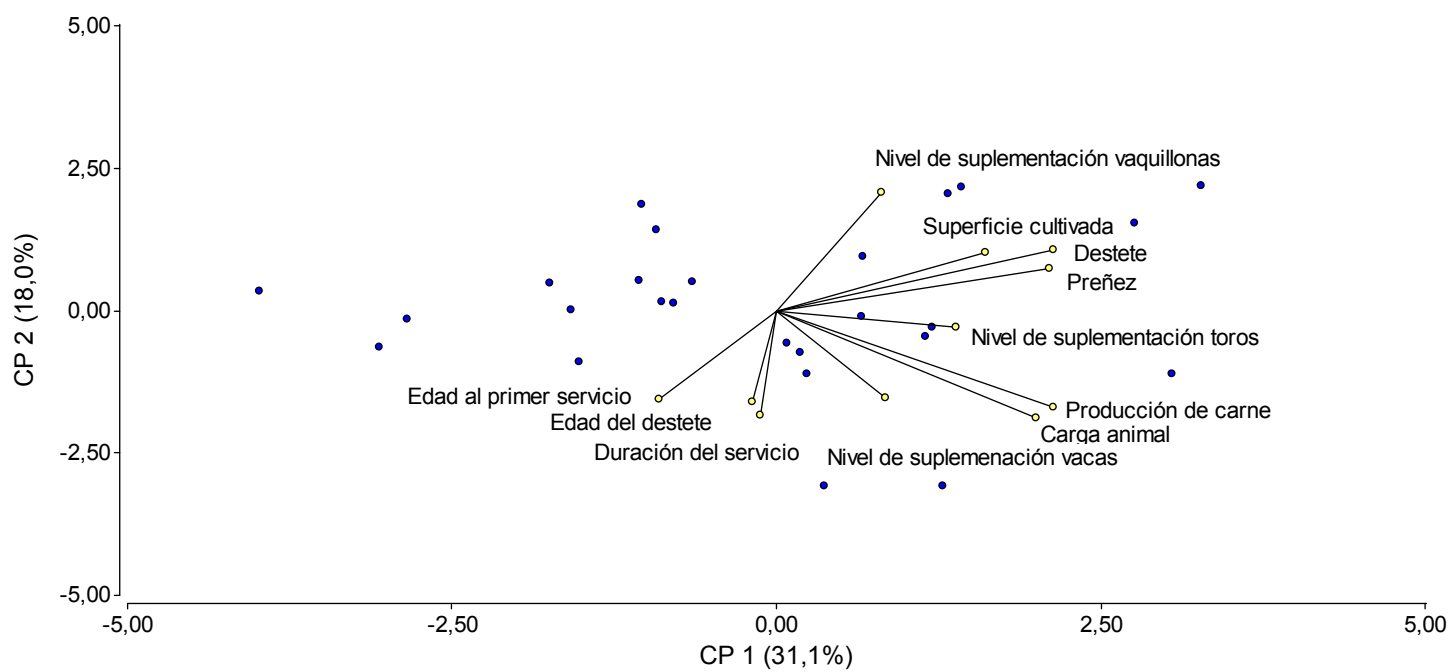

Fig. 1. Gráfico biplot derivado del análisis de componentes principales. Las observaciones (predios de cría) se expresan como puntos y las variables analizadas como líneas rectas: Nivel de suplementación de vaquillonas ( $\%$ del peso vivo); Superficie cultivada ( $\%$ de la superficie de cría - incluye verdeos anuales y praderas megatérmicas); Destete (\%); Preñez (\%); Nivel de suplementación de toros (\% del peso vivo); Producción de carne ( $\mathrm{kg} \mathrm{PV} \mathrm{ha}^{-1}$ año $\left.^{-1}\right)$; Carga animal (vientres $\mathrm{ha}^{-1}$ ); Nivel de suplementación de vacas ( $\%$ del peso vivo); Duración del servicio (meses); Edad del destete (meses); y Edad al primer servicio (meses).

Fig. 1. Biplot graph derived from the principal component analysis. Observations (cow-calf systems) are expressed as points and the variables analyzed as straight lines: Heifer supplementation level ( $\%$ of live weight); Cultivated area ( $\%$ of cow-calf area - includes annual crops and subtropical pastures); Weaning (\%); Pregnancy (\%); Bull supplementation level (\% of live weight); Meat production ( $\mathrm{kg} \mathrm{LW} \mathrm{ha-1}$ per year'-1); Stocking rate (cows ha-1); Cows supplementation level (\% live weight); Breeding season (months); Age at weaning (months); and Age at first service (months).

Tabla 3. Correlación entre variables de interés de los sistemas de cría relevados $(n=27)$.

Table 3. Correlation between variables of interest of cow-calf systems surveyed $(n=27)$.

\begin{tabular}{|c|c|c|c|}
\hline Variable 1 & Variable 2 & $\begin{array}{l}\text { Coeficiente de } \\
\text { Pearson }\end{array}$ & p-valor \\
\hline De eficiencia & Tecnológica & & \\
\hline $\begin{array}{l}\text { Carga animal } \\
\left(\text { vacas ha }^{-1}\right)\end{array}$ & $\begin{array}{l}\text { Nivel de suplementación vacas } \\
\text { (\% del peso vivo) }\end{array}$ & 0,43 & $0,0259^{*}$ \\
\hline Preñez (\%) & $\begin{array}{l}\text { Superficie con especies cultivadas }{ }^{(1)} \\
\text { (\% de superficie de cría) }\end{array}$ & 0,43 & $0,0269^{*}$ \\
\hline Destete (\%) & $\begin{array}{l}\text { Superficie con especies cultivadas }{ }^{(1)} \\
\text { (\% de superficie de cría) }\end{array}$ & 0,52 & $0,0051^{* *}$ \\
\hline De eficiencia & De eficiencia & & \\
\hline $\begin{array}{l}\text { Carga animal } \\
\left(\text { vacas ha }^{-1}\right)\end{array}$ & $\begin{array}{l}\text { Producción de terneros } \\
\left(\mathrm{kg} \text { PV ha- } \mathrm{año}^{-1}\right)\end{array}$ & 0,91 & $<0,0001^{* *}$ \\
\hline $\begin{array}{l}\text { Carga animal } \\
\left(\text { vacas ha }^{-1}\right)\end{array}$ & $\begin{array}{l}\text { Producción de carne } \\
\left(\mathrm{kg} P V \mathrm{ha}^{-1} \mathrm{año}^{-1}\right)\end{array}$ & 0,98 & $<0,0001^{* *}$ \\
\hline
\end{tabular}

${ }^{*} \mathrm{y} *$ Indican valores p menores a 0,05 y 0,01, respectivamente.

(1) Verdeos anuales y praderas megatérmicas perennes. 
Tabla 4. Valores promedio \pm desvío estándar de las variables relevadas para los grupos derivados del análisis de agrupamiento jerárquico.

Table 4. Mean values \pm standard deviation (SD) of variables analyzed for the groups derived from the clustering analysis.

\begin{tabular}{lccc}
\hline Variable & Grupo 1 & Grupo 2 & Grupo 3 \\
\hline $\mathrm{N}$ & 15 & 8 & 4 \\
Superficie de cría (ha totales) & $2479 \pm 2203$ & $588 \pm 459$ & $1938 \pm 1164$ \\
Carga animal (vientres ha-1) & $0,36 \pm 0,11$ & $0,67 \pm 0,18$ & $0,54 \pm 0,15$ \\
Producción de carne (kg PV ha ${ }^{-1}$ año $^{-1}$ ) & $62 \pm 24$ & $116 \pm 35$ & $97 \pm 33$ \\
Preñez (\%) & $74 \pm 10$ & $79 \pm 5$ & $85 \pm 0$ \\
Destete (\%) & $66 \pm 10$ & $70 \pm 9$ & $79 \pm 3$ \\
Superficie cultivada & & & \\
$\quad$ (\% de la superficie de cría) & $7 \pm 9$ & $13 \pm 15$ & $30 \pm 20$ \\
Edad de primer servicio (meses) & $23 \pm 3$ & $24 \pm 1$ & $17 \pm 5$ \\
Duración del servicio (meses) & $7 \pm 1$ & $5 \pm 1$ & $4 \pm 1$ \\
Edad de destete (meses) & & $7 \pm 2$ & $5 \pm 1$ \\
Nivel suplementación vacas & & & \\
$\quad$ (\% del peso vivo) & $0,07 \pm 0,18$ & $0,38 \pm 0,44$ & $0,00 \pm 0,00$ \\
$\quad$ Nivel suplement. vaquillonas (\% del peso vivo) & $0,77 \pm 0,46$ & $0,69 \pm 0,65$ & $1,38 \pm 0,25$ \\
Nivel suplementación toros (\% del peso vivo) & $0,47 \pm 0,30$ & $1,06 \pm 0,32$ & $1,13 \pm 0,25$ \\
\hline
\end{tabular}

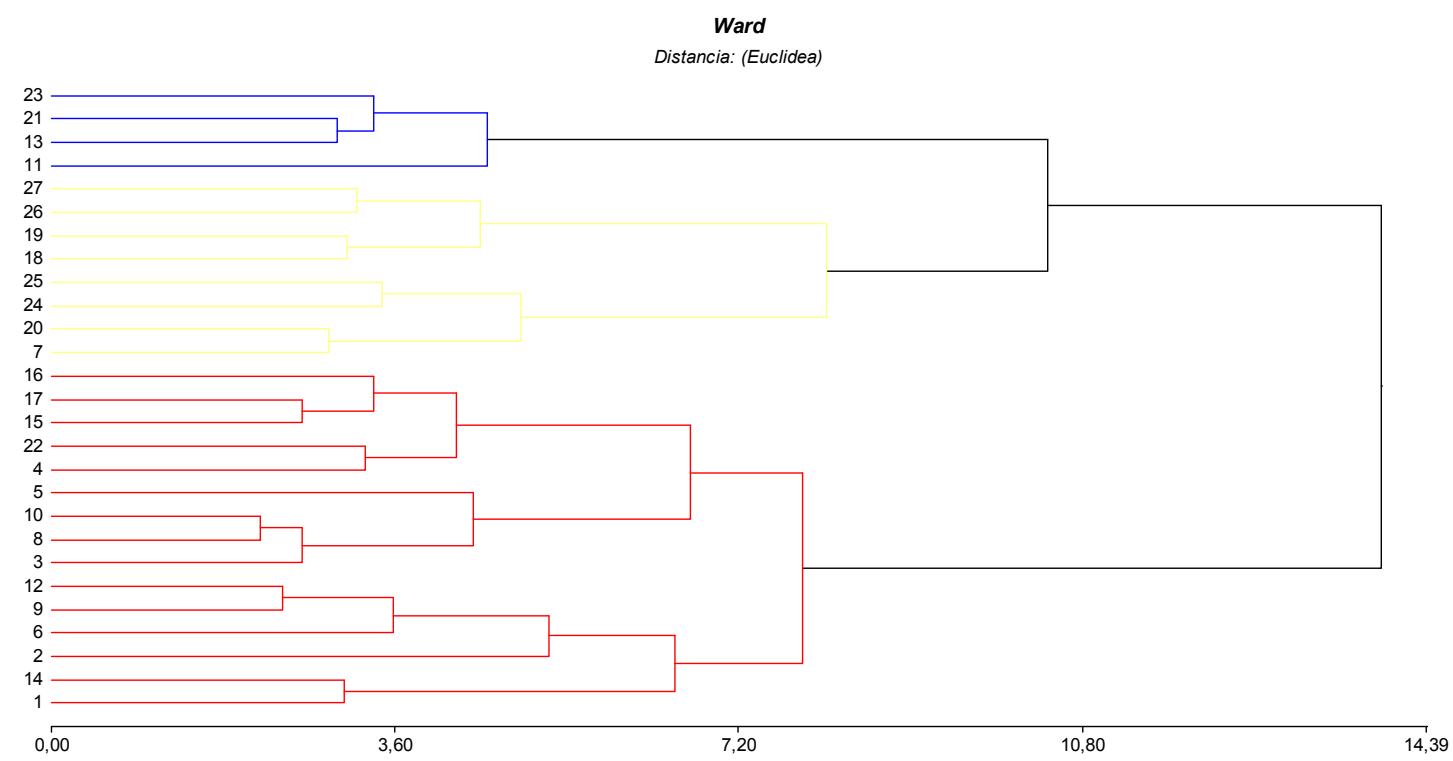

Fig. 2. Dendrograma para los establecimientos relevados derivado del análisis de agrupamiento jerárquico.

Fig. 2. Dendrogram for the surveyed farms derived from the hierarchical grouping analysis.

de tecnologías relacionadas con la nutrición y el manejo reproductivo del rodeo. En este sentido, Dill et al. (2015) encontraron en sistemas de cría bovina de Brasil, que aquellos sistemas que habían adoptado tecnologías relacionadas con la nutrición y la reproducción, como el mejoramiento de praderas naturales, el manejo de la carga animal, la mayor suplementación y la inseminación artificial a tiempo fijo, lograron $83 \%$ de destete, un valor superior comparado a sistemas que no habían incorporado dichas prácticas $(59 \%$ de destete). Por otro lado, es 
importante destacar que los valores de preñez en los sistemas típicos de la región de estudio no se conocen con exactitud por falta de su monitoreo regular (Capozzolo et al., 2017). Sin embargo, acorde a los resultados globales reportados de destete (50\%, MAGyP, 2019), es esperable que la performance reproductiva sea baja y las mermas (pérdidas de terneros entre el tacto y el destete) sean elevadas. Seguramente, los resultados del censo nacional agropecuario de 2018 (CNA, 2018) solo publicados parcialmente, permitirían actualizar y/o confirmar los puntos previos.

Los sistemas de cría típicos de la región presentan bajo nivel de adopción de tecnologías, con suplementación ocasional durante julio y agosto, terneros destetados a los 8 meses de edad en promedio, y vaquillonas con el primer servicio con más de 2 años de edad, y la base de alimentación $100 \%$ en campo natural (Chiossone, 2006; Capozzolo et al., 2017). En este sentido, a partir del análisis de correlaciones entre variables de eficiencia y variables tecnológicas (Tabla 3) y del análisis de componentes principales (Fig.1), se infiere que es posible mejorar la productividad de sistemas ganaderos de cría a partir del incremento de la oferta forrajera (por un aumento de la superficie con especies cultivadas), el aumento de la suplementación de las vacas durante el invierno, y el concomitante aumento de la carga animal. De acuerdo con el análisis de conglomerados, existen diferencias en producción de carne a favor de los establecimientos que aplicaron las tecnologías mencionadas (Tabla 4). Así, se observa en dicha tabla que hubo dos estrategias de intensificación diferentes en el grupo 2 y 3 de conglomerados respecto al grupo 1. En el grupo 2, la mayor producción de carne respecto al grupo 1 estuvo asociada principalmente a una mayor carga animal (vientres ha-1) y a un mayor nivel de suplementación de las vacas (\% del peso vivo). En cambio, en el grupo 3, la mayor producción de carne respecto al grupo 1 estuvo asociada principalmente a mayor superficie cultivada (\% de la superficie de cría), mayor preñez (\%) y mayor destete (\%) y mayor carga animal (Tabla 4). Este análisis permite concluir que si se combinan las principales estrategias de los grupos 2 y 3 , se podría lograr un nivel de producción de carne aún mayor.

En concordancia con los resultados del presente estudio, investigaciones de otras regiones ganaderas de Argentina mostraron que es posible incrementar la producción de carne a partir del aumento de la oferta forrajera, mediante la incorporación de praderas cultivadas, perennes y anuales, combinadas con otras prácticas de manejo. Faverin et al. (2019) reportaron un incremento de entre 17 a $23 \%$ de la producción de carne (kg de PV ha ${ }^{-1}$ año $\left.^{-1}\right)$ a partir del reemplazo de $10 \%$ de la superficie de campo natural con una pradera perenne bajo buenas prácticas de manejo, y de la reducción de la edad de primer servicio de las vaquillonas de 27 a 15 meses. Por otro lado, Fernandez Rosso et al. (2018) registraron $63 \%$ mayor producción de carne cuando se reemplazó el $80 \%$ de la superficie con especies cultivadas (praderas perennes y verdeos anuales), combinando prácticas como el destete precoz de los terneros y reducción de la edad de primer servicio de las vaquillonas. A su vez, los resultados del presente estudio mostraron que la suplementación de las vacas se correlacionó de manera positiva con la carga animal (vacas ha $^{-1}$ ) (Tabla 3). El aumento de la carga animal y mejorando el pastoreo, en simultáneo con el incremento de la cantidad y calidad de praderas, es una estrategia que permite mejorar la productividad de los sistemas de producción de carne por hectárea (Hodgson, 1990; McCarthy et al., 2016), debido a un incremento en la eficiencia de cosecha lo que contribuye a una mayor eficiencia global del sistema (Baudracco et al., 2010). La posibilidad de incrementar la eficiencia de cosecha en 10 o $20 \%$ respecto al promedio regional generaría un fuerte incremento de la productividad.

Aunque existen muchos esfuerzos por incrementar la eficiencia productiva de los productores de la región bajo estudio (Chiossone, 2006; Capozzolo et al., 2017), la falta de datos actualizados para la caracterización de sistemas ganaderos limita el diseño de estrategias de innovación tecnológica regional. En un estudio donde se relaciona productividad e impacto ambiental de sistemas de producción de carne de diferentes regiones de Argentina (Gonzalez Fischer y Bilenca, 2020), se ha reportado que es posible aumentar $15 \%$ la producción de carne a partir de la incorporación de tecnología fácilmente disponible, como las descriptas aquí (Tabla 1 y 2). A los fines de cuantificar el impacto potencial de la adopción del nivel productivo reportado en este trabajo $(+84 \%$ de productividad que los sistemas típicos), es posible inferir que se podrían producir 76.000 terneros extras y la producción regional se incrementaría $21 \%$ si el $25 \%$ de los productores alcanzara el nivel productivo observado en las Tablas 1 y 2. Estos resultados son muy alentadores, pero se requerirá estudios complementarios para identificar potenciales compensaciones productivas y ambientales, como las realizadas para la Pampa Deprimida (Bilotto et al., 2019; Fernandez Rosso et al., 2020). Finalmente, la disponibilidad de la información de este trabajo permitirá avanzar en el diseño de estrategias productivas que tiendan 
a incrementar la producción de carne en una zona de alta relevancia productiva de bovinos de carne de Argentina, como lo es el norte de Santa Fe.

\section{CONCLUSIONES}

Los indicadores productivos y reproductivos relevados en este estudio fueron superiores a los informados para sistemas de cría típicos de la región bajo estudio, debido a una mayor oferta forrajera (por mayor superficie con praderas perennes y verdeos) y al manejo mejorado del rodeo (mayor suplementación de las vacas y mayor carga animal). El análisis de conglomerados permitió observar que hubo un grupo de productores que basó su estrategia de intensificación y mayor producción de carne en mayor carga animal y mayor nivel de suplementación, y otro grupo que basó su estrategia con mayor superficie cultivada, mayor preñez (\%) y mayor destete (\%). Si se combinan ambas estrategias se podría lograr un nivel de producción aún mayor. Los datos relevados serán de utilidad para el estudio y diseño de estrategias de adopción de tecnología que favorezcan la mejora productiva de sistemas de cría del norte de Santa Fe.

\section{RECONOCIMIENTOS}

Los autores desean agradecer en general a los Consorcios Regionales de Experimentación Agrícola región Norte de Santa Fe (CREA) y sus asesores, por proporcionar los datos, colaborar en su discusión con valiosas sugerencias para la descripción de sistemas tecnificados de la región. Este estudio es parte del programa de doctorado en Ciencias Agrarias de la Universidad Nacional del Litoral de la primera autora, y está financiado por CONICET y el proyecto PICT-2017-2271 ("Modelación productiva, económica y ambiental de sistemas modales de producción de carne de base pastoril de la cuenca del Salado y norte de Santa $\left.\mathrm{Fe}^{\prime \prime}\right)$.

\section{LITERATURA CITADA}

Abdala, A.A., M.G. Maciel, E. Salado, R. Aleman, y D. Scandolo. 2013. Pérdidas de preñez en un rodeo de cría del norte de la provincia de Santa Fe. Rev. Arg. Prod. Anim. 33(2):109115.

Baudracco, J., N. Lopez-Villalobos, C.W. Holmes, and K.A. Macdonald. 2010. Effects of stocking rate, supplementation, genotype and their interactions on grazing dairy systems: a review. New Zeal. J. Agr. Res. 53(2):109-133. doi:10.1080/00288231003777665.
Bilotto, B., P. Recavarren, R. Vibart, and C.F. Machado. 2019. Backgrounding strategy effects on farm productivity, profitability and greenhouse gas emissions of cow-calf systems in the Flooding Pampas of Argentina. Agricultural Systems 176:1-15. doi: 10.1016/j. agsy.2019.102688.

Britt, J.H., R.A. Cushman, C.D. Dechow, H. Dobson, P. Humblot, M.F. Hutjens, et al.. 2018. Invited review: Learning from the future-A vision for dairy farms and cows in 2067. J. Dairy Sci. 101:3722-3741. doi: 10.3168/ jds.2017-14025.

Canosa, M.R. 2003. Indicadores de eficiencia de la cría. Cuaderno de Actualización Técnica No 66: Cría Vacuna. AACREA, Buenos Aires, Argentina.

Capozzolo, C., J. Scarel, M.E. Ocampo, R. Ybran, O. Hug, y P. Mitre. 2017. Sistemas ganaderos bovinos - Caracterización del distrito Toba. Ediciones INTA. 1 ${ }^{a}$. ed. INTA Reconquista, Reconquista, Argentina. Disponible en https://inta.gob.ar/documentos/sistemasganaderos-bovinos-caracterizacion-deldistrito-toba. (Consulta 17 abril 2020)

CNA. 2008. Censo Nacional Agropecuario 2008. Disponible en https://www.indec.gob.ar/ indec/web/Nivel4-Tema-3-8-87 (Consulta 15 abril 2020).

CNA. 2018. Censo Nacional Agropecuario 2018. Resultados Preliminares. Disponible en https://cna2018.indec.gob.ar/ (Consulta 15 abril 2020).

Chimicz, J. 2006. Tipificación de la cría bovina en Santa Fe. Una propuesta para la elaboración de estrategias diferenciales de extensión. INTA EEA Rafaela, Rafaela, Argentina. Disponible en https://inta.gob.ar/sites/ default/files/script-tmp-tipificacin_de_la cra_bovina_en_santa_fe.pdf (Consulta 17 abril 2020).

Chiossone, G. 2006. Sistemas de producción ganaderos del noreste argentino: Situación actual y propuestas tecnológicas para mejorar su productividad. En X Seminario de manejo y utilización de pastos y forrajes en sistemas de producción animal. 20-22 de Abril. Maracaibo, Venezuela. Universidad del Zulia, FCV, Zulia, Venezuela.

Dhein Dill, M., G. Ribas Pereira, J. Gonçalves Costa Jr, L. Canali Canellas, V. Peripolli, J. Braccini Neto, et al. 2015. Technologies that affect the weaning rate in beef cattle production systems. Trop. Anim. Health Pro. 47(7):1255-1260. doi: 10.1007/s11250-0150856-х. 
Di Rienzo, J.A., F. Casanoves, M.G. Balzarini, L. Gonzalez, M. Tablada, y C.W. Robledo. 2011. InfoStat versión 2011. Grupo InfoStat. FCA, Universidad Nacional de Córdoba, Argentina. Disponible en http://www. infostat.com.ar. (Consulta 17 abril 2020)

FAO 2019. How to Feed the World in 2050. Available at http://www.fao.org/fileadmin/ templates/wsfs/docs/Issues_papers/ HLEF2050_Global_Agriculture.pdf (Accessed 14 April 2020).

Faverin, C., F. Bilotto, C. Fernández Rosso, y C. Machado. 2019. Modelación productiva, económica y de gases de efecto invernadero de sistemas típicos de cría bovina de la Pampa Deprimida. Chilean J. Agric. Anim. Sci. 35(1):14-25.

Faverin, C., y C.F. Machado. 2019. Tipologías y caracterización de sistemas de cría bovina de la Pampa Deprimida. Chilean J. Agric. Anim. Sci. 35(1):3-13.

Fernández Rosso, C., F. Bilotto, A. Lauric, G.A. De Leo, C. Torres Carbonell, M.A. Arroqui, et al. 2020. An innovation path in Argentinean cow-calf operations: Insights from participatory farm system modelling. Syst. Res. Behav. Sci. 1-15. doi: 10.1002/sres.2679.

Fernandez Rosso, C., M. Lauric, G. De Leo, F. Bilotto, C. Torres Carbonell, y C. Machado. 2018. Modelación productiva, económica y emisión de metano en sistemas de cría vacuna de Bahía Blanca y Coronel Rosales. Rev. Inv. Agrop. 44(2):129-135.

Gallacher, M., and D. Lema. 2018. Returns to managerial ability and technical efficiency in Argentina dairy farms. In International Association of Agricultural Economists (IAAE). July 28-August 2. Vancouver, British Columbia, Canadá. doi:10.22004/ ag.econ.277323.

Giorgi, R., R. Tosolini, V. Sapino, J. Villar, C. León, y A. Chiavassa. 2007. Zonificación agroeconómica de la Provincia de Santa Fe. Delimitación y descripción de las zonas y subzonas agroeconómicas. Publicación Miscelánea $N^{\circ} 110$. INTA EEA Rafaela, Instituto Nacional de Tecnología Agropecuaria, Buenos Aires, Argentina.

Gonzalez Fischer, C., and D. Bilenca. 2020. Can we produce more beef without increasing its environmental impact? Argentina as a case study. Perspect. Ecol. Conserv. 18:1-11. doi:10.1016/j.pecon.2019.12.002.

Hodgson, J. 1990. Grazing Management: Science into Practice. 203 p. Longman Handbooks in Agriculture. Longman Scientific and Technical co-published with John Wiley, New York, USA.
INTA. 2018. Estación Meteorológica Reconquista. Instituto Nacional de Tecnología Agropecuaria. Disponible en https://inta. gob.ar/documentos/estacion-meteorologicareconquista (Consulta 15 abril 2020).

IPCVA. 2016. Potencial productivo de la ganadería bovina de la provincia de Santa Fe. Instituto de Promoción de la Carne Vacuna Argentina, Buenos Aires, Argentina. Disponible en http://www.ipcva.com.ar/ vertext.php?id=1620 (Consulta 17 abril 2020).

Kuivanen, K.S., M. Michalscheck, K. Descheemaeker, S. Adjei-Nsiah, S. MellonBedi, J.C.J. Groot, et al. 2016. A comparison of statistical and participatory clustering of smallholder farming systems - A case study in Northern Ghana. J. Rural Stud. 45:184-198. doi: 10.1016/j.jrurstud.2016.03.015.

Lara, R., B. Lazzarini, y J. Baudracco. 2019. Caracterización técnico-productiva de fincas lecheras del Noreste de la provincia de Buenos Aires, Argentina. Chilean J. Agric. Anim. Sci. 35(2):186-195.

MAGyP. 2019. Informes Técnicos y Estimaciones. Ministerio de Agricultura Ganadería y Pesca de Argentina, Buenos Aires, Argentina. Disponible en https://www.agroindustria. gob.ar (Consulta 14 abril 2020).

McCarthy, B., L. Delaby, M. Pierce, J. McCarthy, C. Fleming, A. Brennan, et al. 2016. The multi-year cumulative effects of alternative stocking rate and grazing management practices on pasture productivity and utilization efficiency. J. Dairy Sci. 99:37843797. http://dx.doi.org/10.3168/jds.2015-9763.

Meat and Livestock Australia. 2017. How are global and Australian beef producers performing? Global agri benchmark network results 2016. Available at https://www.mla. com.au/globalassets/mla-corporate/prices-markets/documents/trends--analysis/ agri-benchmark/mla_agribenchmark-beefresults-report_jan-2018.pdf (Accessed 14 April 2020).

Pacín, F., and M. Oesterheld. 2015. Closing the technological gap of animal and crop production through technical assistance. Agric. Syst. 137:101-107. doi: 10.1016/j. agsy.2015.04.007.

Pighin, D., A. Pazos, V. Chamorro, F. Paschetta, S. Cunzolo, F. Godoy, et al. 2016. A contribution of beef to human health: A review of the role of the animal production systems. Sci. World J. 2016:1-10. doi: 10.1155/2016/8681491. 
Rearte, D.H., and A.J. Pordomingo. 2014. The relevance of methane emissions from beef production and the challenges of the Argentinean beef production platform. Meat Sci. 98:35-360. doi: 10.1016/j. meatsci.2014.06.021.

USDA. 2019. Livestock and products semi-annual, Australia. United States Department of Agriculture - GAIN Report AU1902. Available at https://apps.fas.usda.gov/newgainapi/api/report/wnloadreportbyfilename?filename $=$ Livestock $\% 20$ and $\% 20$ Products $\% 20$ Semi-annual_Canberra_Australia_3-1-2019. pdf (Accessed 14 April 2020).

Ward, J.H. 1963. Hierarchical grouping to optimize an objective function. J. Am. Stat. Assoc. 58:236-244. 